\title{
Functional segmentation of the hippocampus in the healthy human brain and in Alzheimer's disease
}

\author{
Mojtaba Zarei a,*, Christian F. Beckmann ${ }^{\text {b }}$, Maja A.A. Binnewijzend ${ }^{\mathrm{c}}$, Menno M. Schoonheim ${ }^{\mathrm{c}}$, \\ Mohammad Ali Oghabian ${ }^{\mathrm{d}}$, Ernesto J. Sanz-Arigita ${ }^{\mathrm{c}}$, Philip Scheltens ${ }^{\mathrm{e}}$, Paul M. Matthews ${ }^{\mathrm{f}}$, Frederik Barkhof ${ }^{\mathrm{c}}$ \\ a Institute for Cognitive Sciences Studies, Tehran, I.R., Iran \\ ${ }^{\mathrm{b}}$ Donder Institute, Radbound University, Nijmegen, Netherlands \\ c Radiology and Image Analysis Centre, VU Medical Centre, Amsterdam, Netherlands \\ ${ }^{\mathrm{d}}$ Department of Neurology and Alzheimer Center, VU Medical Center, Amsterdam, Netherlands \\ e Institute for Advanced Medical Technologies, Tehran University of Medical Sciences, Tehran, Iran \\ ${ }^{f}$ Center for Neuroscience, Imperial College London, London, UK
}

\section{A R T I C L E I N F O}

Article history:

Accepted 1 October 2012

Available online 3 November 2012

\begin{abstract}
A B S T R A C T
In this study we segment the hippocampus according to functional connectivity assessed from resting state functional magnetic resonance images in healthy subjects and in patients with Alzheimer's disease (AD). We recorded the resting FMRI signal from 16 patients and 22 controls. We used seed-based functional correlation analyses to calculate partial correlations of all voxels in the hippocampus relative to characteristic regional signal changes in the thalamus, the prefrontal cortex (PFC) and the posterior cingulate cortex (PCC), while controlling for ventricular CSF and white matter signals. Group comparisons were carried out controlling for age, gender, hippocampal volume and brain volume. The strength of functional connectivity in each region also was correlated with neuropsychological measures.

We found that the hippocampus can be segmented into three distinct functional subregions (head, body, and tail), according to the relative connectivity with PFC, PCC and thalamus, respectively. The AD group showed stronger hippocampus-PFC and weaker hippocampus-PCC functional connectivity, the magnitudes of which correlated with MMSE in both cases.

The results are consistent with an adaptive role of the PFC in the context of progression of dysfunction in PCC during earlier stages of AD. Extension of our approach could integrate regional volume measures for the hippocampus with their functional connectivity patterns in ways that should increase sensitivity for assessment of $\mathrm{AD}$ onset and progression.
\end{abstract}

(c) 2012 Elsevier Inc. All rights reserved.

\section{Introduction}

Studies in animals show that the hippocampus can be anatomically and functionally segmented into distinct subregions along its ventrodorsal axis in rat which is equivalent to the anterio-posterior axis in primates (Fanselow and Dong, 2010). Human studies suggest an anterior-posterior subdivision of cognitive functional domains in the hippocampus (Dong et al., 2009; Greicius et al., 2003a; Maguire et al., 1997; Moser and Moser, 1998).

Anatomical studies using anterograde or retrograde neural tracing have demonstrated that the hippocampus has direct connections with the anterior and ventromedial nuclei of the thalamus in both rodents and primates (Amaral and Cowan, 1980; Parvizi et al., 2006; van Groen et al., 1999). We previously demonstrated similar connections in

\footnotetext{
* Corresponding author at: Institute for Cognitive Sciences Studies, Vali-e-Asr Street, Tehran, 1594834111, I.R., Iran.

E-mail address: mzarei@me.com (M. Zarei).
}

human brain using probabilistic diffusion tensor tractography (Zarei et al., 2010). The connection of the hippocampus with PFC via the parahippocampus and entorhinal cortex in monkey has been also reported using invasive neural tracing techniques (Carmichael and Price, 1995; Insausti et al., 1987; Lavenex et al., 2002; Morris et al., 1999; Suzuki and Amaral, 1994). The posterior cingulate cortex (Lavenex et al., 2002; Morris et al., 1999; Parvizi et al., 2006; Suzuki and Amaral, 1994; van Groen et al., 1999) and posterior parietal cortex (Lavenex et al., 2002; Mesulam et al., 1977) also are connected with hippocampus via parahippocampal gyrus and entorhinal cortex.

FMRI studies have identified several so-called resting state networks (RSNs), defined as brain regions displaying high temporal correlations in spontaneous changes of the BOLD signal of the unstimulated, resting brain (Beckmann et al., 2005; Damoiseaux et al., 2009; Fox et al., 2005; Raichle, 2010). One well-known network is known as the "default mode network" (DMN) (Biswal et al., 1995; Cordes et al., 2001) which includes the PCC, precuneus and anterior cingulate, medial prefrontal and lateral parietal cortices as well as the hippocampus and thalamus (Greicius et al., 2009). The DMN is characterized by correlated BOLD 
signal reductions during the performance of cognitive tasks relative to the resting state (Greicius et al., 2003b, 2004; Raichle et al., 2001).

A resting state functional connectivity study revealing two separate brain networks correlated with distinct subregions within the medial temporal region suggested that connectivity might be able to be used to functionally parcellate the hippocampus (Kahn et al., 2008). These authors showed that activity in the body of hippocampus and posterior parahippocampal gyrus correlated with the activity in lateral parietal cortex, posterior cingulate, retrosplenial cortex, and ventral medial prefrontal cortex, while resting BOLD signal in the anterior hippocampus and the perirhinal/entorhinal cortices correlated with the lateral temporal cortex extending into the temporal pole. Functional parcellation of the human thalamus (Zhang et al., 2008) and cerebellum (O'Reilly et al., 2009) has been possible using these methods.

Such work may be relevant to relating structural, functional and behavioral pathologies in AD. Several studies have shown changes of functional connectivity between structures that are part of the DMN in earlier stages of AD (Greicius et al., 2004; Sorg et al., 2007; Wang et al., 2006; Zhang et al., 2009). For example, reduced functional connectivity between the right hippocampus and medial PFC, ventral anterior cingulate cortex and PCC, as well as an increase of functional connectivity between the left hippocampus and the right lateral PFC were reported (Stein et al., 2000; Wang et al., 2006).

In this study we tested: 1) if the human hippocampus can be segmented into distinct subregions based on relative patterns of functional connectivity in the "resting state"; 2 ) if connectivity pattern of the hippocampal subregions changes in $\mathrm{AD}$; and, 3 ) if the magnitude of change in regional functional connectivity of the hippocampus can be related with cognitive impairment in AD.

\section{Methods}

Subjects

MR images were obtained from two groups of right-handed subjects: 22 old healthy subjects without memory complaints (age $70.7 \pm$ 6.0 , range 60 to 81 years; MMSE $28.7 \pm 1.4 ; 13$ females), and 16 patients with mild Alzheimer's disease (AD) (age 69.5 \pm 6.9 years, range 59 to 79 years; MMSE 22.9 $\pm 3.2 ; 8$ females). Patients were recruited at the Alzheimer Center of the VU University Medical Center, Amsterdam, the Netherlands. Diagnostic criteria of AD were that of NINCDSADRDA (McKhann et al., 1984), with MMSE scores $>18$ and $C D R<2$. Healthy subjects were recruited by two means: (1) as family members of patients and (2) through advertisements posted in the medical center, the medical faculty of the University and activity centers for the elderly in the community.

The Ethical Review Board of the VU Medical Center, Amsterdam approved the study. Informed consent was provided by the all participants or his/her legal guardian. Participants were excluded if they had any significant medical, neurological or psychiatric illness (other than AD in the patient group) or if they were taking medication known to influence cerebral function (except for AD medication such as cholinesterase inhibitors). In order to assess the presence of vascular abnormalities in the white matter, T2-weighted fluid attenuation inversion recovery (FLAIR) scans of each subject were reviewed by a neuroradiologist. Subjects with WM abnormalities outside the normal range were excluded from participation in the study. The Fazekas scale provides a summary index of white matter hyperintensities for the entire brain as assessed from T2-weighted images. Mean Fazekas score (Fazekas et al., 1987) for control group was zero and for AD group $0.9 \pm 0.9$ (mean $\pm S D$ ).

\section{Neuropsychological assessment}

All participants underwent a MMSE, Geriatric Depression Scale (GDS) and the Dutch version of the New Adult Reading test (NLV) as an indicator of premorbid IQ followed by an extensive neuropsychological test
Table 1

Patient characteristics and neuropsychological profile.

\begin{tabular}{|c|c|c|c|}
\hline & Control & $A D$ & $\begin{array}{l}\text { AD versus } \\
\text { control }\end{array}$ \\
\hline & $M \pm \mathrm{SD}$ & $M \pm \mathrm{SD}$ & $\mathrm{t}$ \\
\hline Gender (male/female) & $9 / 13$ & $8 / 8$ & 0.54 \\
\hline Age (years) & $70.73 \pm 6.0$ & $69.50 \pm 6.7$ & 0.60 \\
\hline MMSE & $28.73 \pm 1.4$ & $22.88 \pm 3.2$ & $6.78^{*}$ \\
\hline GDS & $0.64 \pm 1.0$ & $1.20 \pm 1.2$ & 1.51 \\
\hline NLV & $110.67 \pm 18.7$ & $102.5 \pm 19.7$ & 1.29 \\
\hline
\end{tabular}

Attention, concentration and speed

Digit span

Backward span

WAIS Symbol

substitution/encoding

Trail making test $\mathrm{A}$

Stroop

Word card

Color card

Color-word card

$5 \pm 1.4$

$3.87 \pm 1.0$

$\begin{array}{ll}58.73 \pm 15.3 & 36.80 \pm 21.4\end{array}$

3.07

4.07

3.65

$43.55 \pm 15.4 \quad 72.20 \pm 29.0$

3.50

$46.18 \pm 6.4 \quad 54.69 \pm 13.6$

$60.82 \pm 11.0 \quad 84.69 \pm 26.0$

$116.73 \pm 37.3 \quad 180.27 \pm 72.8$

2.32

3.46

2.94

Episodic memory

15 word test

Total immediate recall

Delayed recall

Visual association test

A

B

WAIS symbol

substitution/memory

Cued reproduction

Free reproduction

Memory impairment

screen plus

Direct reproduction

Delayed reproduction

$42.91 \pm 10.7$
$8.41 \pm 3.1$
$11.50 \pm 1.1$
$10.63 \pm 1.5$

$21.88 \pm 5.9$

$1.63 \pm 1.7$

$7.75^{*}$

$8.57^{*}$

$5.13 \pm 3.8$

$3.06 \pm 3.6$

$6.47^{*}$

$4.85^{*}$

$9.40 \pm 4.0$

$2.46 \pm 2.8$

$5.37^{*}$

$6.41 \pm 1.6$

$3.08 \pm 1.5$

$6.19^{*}$

$10.95 \pm 1.6$

$8.80 \pm 2.5$

$2.85 \pm 2.7$

3.40

$9.67^{*}$

Executive function

WISC maze

Total time (s)

Mistakes

Trail making test $\mathrm{B}$

Fluency

Animals (within $2 \mathrm{~min}$ )

Insects (within $1 \mathrm{~min}$ )

$217.50 \pm 87.8$

$2.45 \pm 1.6$

$299 \pm 147.1$

2.12

$101.09 \pm 44.2$

$4.58 \pm 4.1$

1.91

$35.23 \pm 7.9$

$18.27 \pm 6.7$

4.26

$8.95 \pm 3.1$

$4.67 \pm 2.4$

$6.82^{*}$

$4.50^{*}$

Praxis

Rey complex figure

Copy

$32.71 \pm 3.0$

$25.88 \pm 8.6$

2.76

Organization

$3.33 \pm 2.0$

$1.92 \pm 1.9$

2.00

MMSE $=$ mini mental status examination, GDS $=$ geriatric depression scale, $\mathrm{NLV}=$ the Dutch version of the New Adult Reading test WAIS = Wechsler adult intelligence scale, WISC $=$ Wechsler intelligence scale for children, TMT $=$ trail making test. Note that higher scores in Stroop, WISC maze, and MMSE indicate poorer performance. * indicates significant difference at $\mathrm{p}=0.0005$.

battery including tests measuring attention/concentration, processing speed, episodic memory, executive functioning and praxis (see Table 1 for details). Scores on these neuropsychological tests were compared between groups using independent t-tests (one-tailed, control $>A D$ ). The significance threshold was set at $\mathrm{p}<0.05$ after Bonferroni correction for multiple comparisons.

\section{Image acquisitions}

Magnetic resonance imaging (MRI) examinations were conducted on a 1.5 T Sonata system (Sonata, Siemens AG, Erlangen, Germany), and included a T1-weighted 3D MR sequence (TR $=2700 \mathrm{~ms}$, TE $=$ $3.97 \mathrm{~ms}, \mathrm{TI}=950 \mathrm{~ms}$, flip angle $=8^{\circ}, 160$ coronal slices, voxel size $=$ $1 \times 1.5 \times 1 \mathrm{~mm}$ ). Head motion was minimized by the use of tightly padded clamps attached to the head coil. 
For resting state FMRI, an echo planar imaging sequence was used $\left(\mathrm{TR}=2850 \mathrm{~ms}, \mathrm{TE}=60 \mathrm{~ms}\right.$, flip angle $90^{\circ}$, matrix $64 \times 64$, field of view $192 \times 192 \mathrm{~mm}$,), to obtain 200 volumes with isotropic $3.0 \mathrm{~mm}$ voxels. Subjects were asked to stay awake during the scan.

\section{Data preprocessing}

Data processing was carried out using tools from the FMRIB Software Library, FSL 4.1 (Smith et al., 2004). The first 6 volumes from the functional MRI runs were discarded, then the following pre-statistic processing was applied: motion correction using MCFLIRT (Jenkinson and Smith, 2001), spatial smoothing using a Gaussian kernel of FWHM $6 \mathrm{~mm}$, grand-mean intensity normalization of the entire 4D data set by a single multiplicative factor, high-pass temporal filtering (Gaussian-weighted least-squares straight line fitting, with sigma= $50.0 \mathrm{~s}$ to ensure that at least half of power was retained for frequencies down to $0.01 \mathrm{~Hz}$.

\section{Masks}

We initially conducted a pilot study and found that hippocampal segments showed functional connectivity with the precuneus and the posterior parietal and posterior cingulate cortices but that the hippocampal connectivities overlap and do not distinguish subregions. Similarly, hippocampal segments that were functionally connected with PFC subregions (dorsolateral, orbitofrontal, frontal polar and ventrolateral cortex) also overlap. However, functional connectivities to the prefrontal and posterior cingulate cortices and to the thalamus showed anatomically distinct patterns of in the hippocampus. These then were characterized in the work described in this report.

The thalamus, hippocampus, prefrontal and posterior cingulate masks (spatially normalized to the Montreal Neurological Institute (MNI) brain template) were taken from the Harvard-Oxford Structural Atlas (http:// www.fmrib.ox.ac.uk/fsl/data/atlas-descriptions.html), available as a toolbox in FSLview (http://www.fmrib.ox.ac.uk/fsl/fslview). These cortical masks were transformed into the space of individual anatomical scans using FNIRT, FMRIB's Nonlinear Image Registration Tool (Andersson et al., 2007), and then into individual functional space using FLIRT, FMRIB's linear image registration tool (Jenkinson and Smith, 2001). These masks were individually checked to ensure accuracy of the masks created and

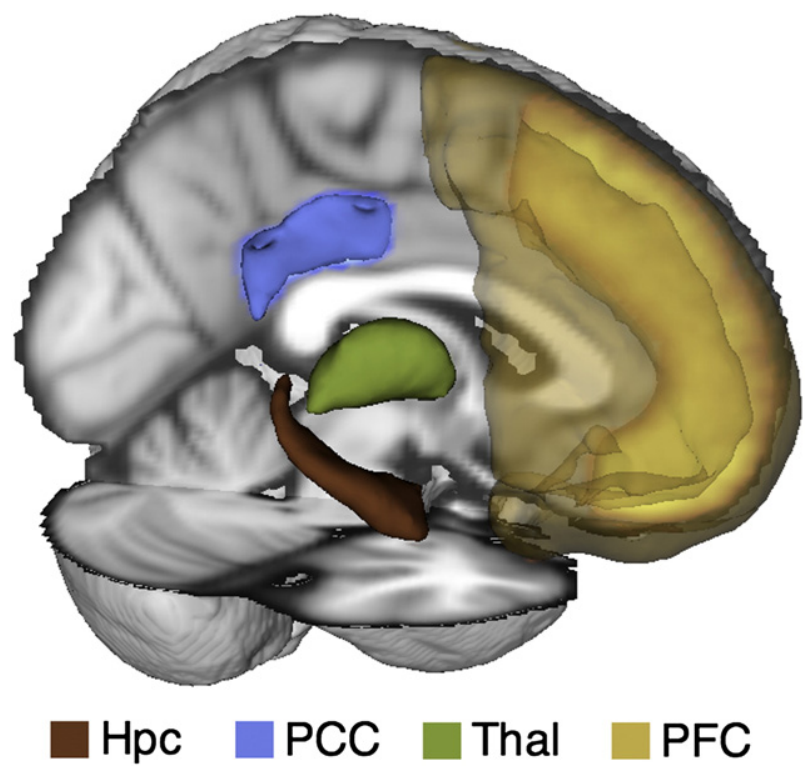

Fig. 1. Shows seed and target masks including prefrontal cortex (PFC), posterior cingulate cortex (PCC), thalamus (Thal) and hippocampus (Hpc). registered to functional space using FLIRT before functional connectivity was calculated. Fig. 1 shows all masks.

Voxel-wise correlation mapping

We used a voxel-wise approach to map resting state functional connectivity across the hippocampus, between each voxel in the hippocampal mask and characteristic time series associated with the defined target masks (thalamus, PFC, and PCC). Details of this method have been previously described (O'Reilly et al., 2009). In contrast to the use of independent component analysis in which functional connectivity between different brain regions is explored (Beckmann and Smith, 2004), seed-based functional connectivity analysis requires clear hypothesis and a priori definition of regions of interest (seed regions and target regions) accordingly. In brief, first, we calculated the major Eigen time series representing activity in each of the target masks of thalamus, PFC and PCC in each of the individual subjects. The major Eigen time series is the single time series which best reflects activity across the target mask in that it represents the largest amount of variance across the set of voxels within the mask. In order to discount nuisance effects we also calculated the major Eigen time series in masks representing the white matter and cerebro-spinal fluid (CSF: across the whole brain volume). These masks were first derived using FAST, FSL tissue segmentation tool (Zhang et al., 2001) and similar to all other target masks transformed into the native space of the functional data. Furthermore, time series representing head motion were extracted using MCFLIRT (Jenkinson and Smith, 2001). These 8 confound time series (white matter and CSF Eigen-timeseries plus 6 time series representing head motion) were partialled out of all hippocampal voxel time series and target masks Eigen-time series as part of the partial regression analysis (O'Reilly et al., 2009). The reason for adding confound regressors such as white matter and CSF time series is to minimize the potential confounding effect of including non-gray matter voxels in hippocampus and the target masks, and to remove any non-tissue-specific confound effects such as scanner drift.

Taking the preprocessed functional data, for each voxel in the hippocampus we calculated the partial correlation scores between its BOLD time series and the major Eigen time series for each cortical region, in each case regressing out the time series of all other cortical regions, so that the resulting correlation maps represented only correlations specific to the target mask in question. The output of the analysis took the form of correlation maps (across the hippocampus) in individual functional space, pertaining to each target mask. Finally, voxels were thresholded at $<0.2$ to exclude voxels with weak correlations before group comparison analysis was carried out.

To create an anatomical map that represents the relative likelihood of functional connectivity of each voxel in the hippocampus, each hippocampal voxel was assigned to one of targets with which it had the highest connectivity strength ('hard segmentation').

\section{Group analysis}

Individual un-thresholded correlation maps were registered into MNI space, first using an affine transformation from the individual functional space to the anatomical space, then into MNI space using nonlinear registration implemented using FNIRT (as above). The resulting hippocampal standard-space correlation maps (one per subject and per target mask) were normalized so that the value of each voxel representing the strength of functional connectivity $(r)$ of the voxel with the target masks. These correlational maps were thresholded at 0.2 to remove spurious correlations. A group map of each hippocampus was created for each ipsilateral target mask by averaging $r$ values for all subjects within each group. These maps were assessed for within and between-group effects for hippocampal subregion using nonparametric permutation testing (Randomize, part of FSL) (Nichols and Holmes, 2002) controlling for age, gender, brain and hippocampal 
AD

Rt.
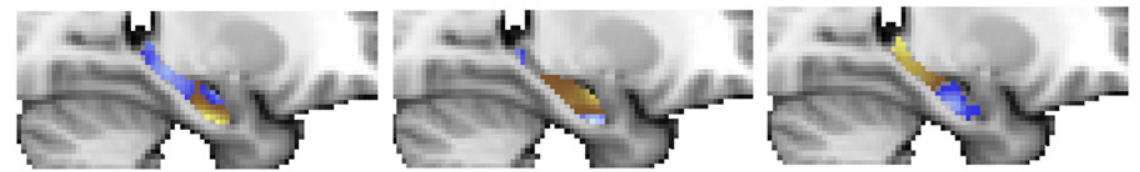

Lt.
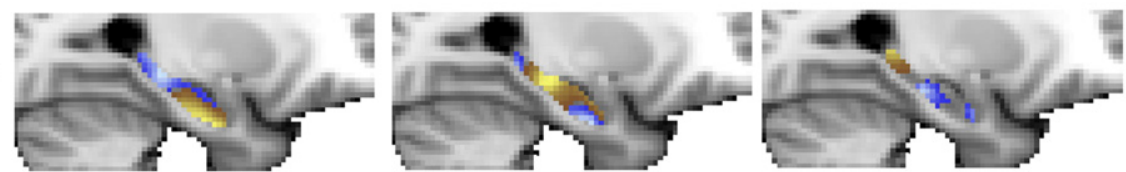

Control

Rt.
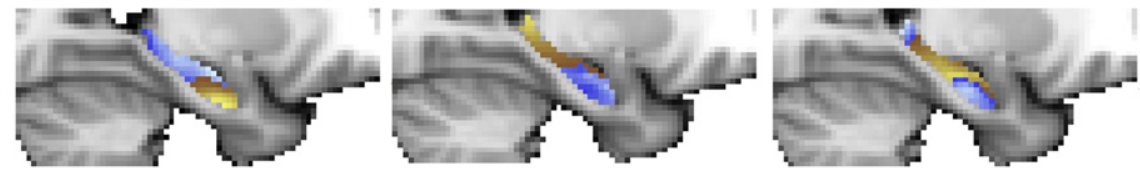

Lt.

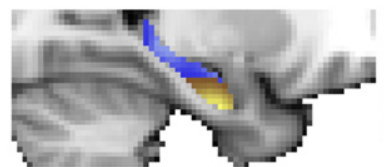

Prefrontal

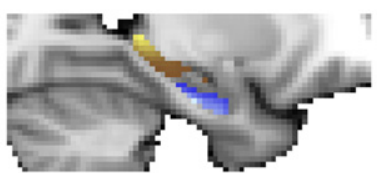

Thalamus

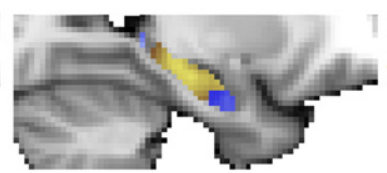

Posterior cingulate

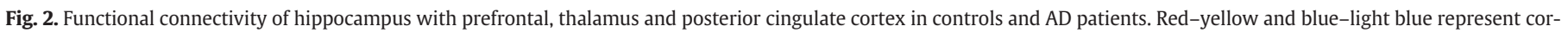
relation and anticorrelation respectively.

volume. The latter is particularly important to ensure that any group difference is not driven by the structural changes in the hippocampus that is often associated with AD. Further correlational analyses were carried out between each functional connectivity map and neuropsychological measures using Pearson's correlation. All results were corrected for voxel-wise multiple comparison using false discovery rate $(\mathrm{p}<0.05)$ (Genovese et al., 2002).

\section{Results}

Demographic and neuropsychological data of patients and control groups have previously been reported (Damoiseaux et al., 2009). Both groups had similar demographic characteristics in terms of age, gender and educational level. As expected, AD subjects performed significantly worse in neuropsychological tests than the control group (see Table 1).

\section{Functional segmentation of the hippocampus in controls}

Seed-based functional connectivity analysis in the healthy control group showed that the hippocampus is differentially connected with the PFC, thalamus, and PCC. Activity of the head of the hippocampus positively correlated with the PFC. A similar subregion of the hippocampus displayed negative correlations with the thalamus and PCC (Fig. 2). Activity in a middle region corresponding to the body of the hippocampus positively correlated with the PCC and negatively correlated with the PFC. Signal changes in the tail of the hippocampus correlated positively with thalamus and PCC (Fig. 2). The few voxels in the tail (which is the smallest part) of hippocampus appeared to be correlated negatively with PFC (color coded in blue).

These observations can be used to create exclusive or "hard segmentation" maps to visualize hippocampal subregions defined on the basis of their strongest functional connectivity to one of the three large ROIs using data from healthy aged subjects. This approach defines three subregions in the longitudinal axis corresponding to the head, body and tail of the hippocampi (Fig. 3). These regions are symmetrically located in both hippocampi, but somewhat different in location depending on whether positive or negative correlations are used for the segmentation (data not shown).

\section{Differences between controls and $A D$ patients}

Good correspondences of subregions of the hippocampi and their predominant functional connectivities were found between data
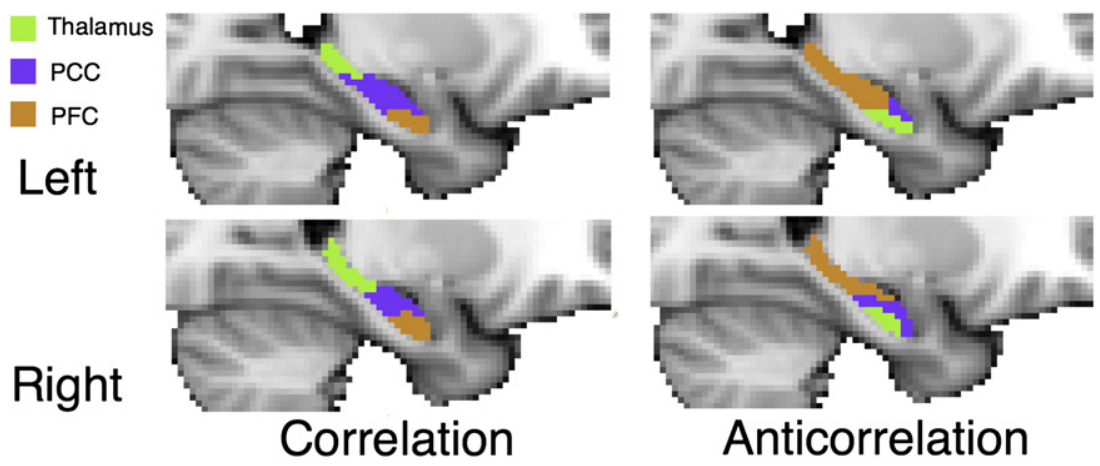

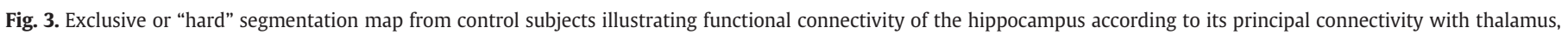
prefrontal or posterior cingulate cortex. 

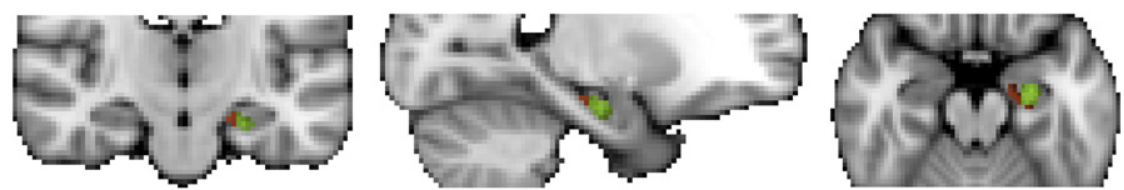

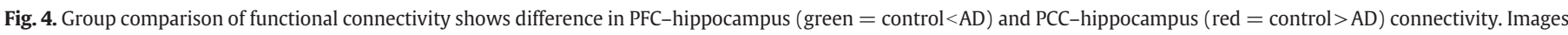
are radiologically oriented.

obtained from the AD and the healthy subject group. Quantitative comparisons of the partial correlations between the groups showed that the mean correlation of the head of the hippocampus with the PFC was stronger in $\mathrm{AD}$ subjects than in controls. In contrast, correlation of the body of the hippocampus with PCC was stronger in the healthy control subjects than in $\mathrm{AD}$ subjects (Fig. 4, Table 2).

To understand the potential behavioral relevance of these differences, the relation between the strength of these partial correlations and behavioral measures was explored. In the AD group, positive partial correlations between the PFC ROI and the head of hippocampus were negatively correlated with MMSE, Memory Impairment Screening (left), Visual Association Test (right), 15 Word Test (left), but positively correlated with Stroop Test (bilateral). In addition, in the AD group positive partial correlations between functional connectivity of the body of hippocampus with the PCC were positively correlated with MMSE (right) and 15 Word Test and negatively correlated with the Stroop Test (right) (Figs. 5 and 6).

\section{Discussion}

In this study, we have shown that the hippocampus can be functionally divided into three distinguishable subregions (head, body and tail) according to the dominant functional connectivity in resting state fMRI data with large grey matter ROIs defined anatomically to include the PFC, PCC and thalamus, respectively. To the best of our knowledge this is the first report of anatomical segmentation of the hippocampus based on functional connectivities to other brain regions in humans in vivo.

A population map of the voxel-wise hippocampal functional connectivities to these larger ROIs showed a remarkable consistency across cases. Significant functional connectivities could be found between a subregion defining the head of the hippocampus and the PFC, thalamus and PCC, the body of the hippocampus and the PCC and PFC, and the tail is connected with the thalamus, PCC and PFC. The magnitude and distribution of the partial correlations defining the functional connectivity to the selected target ROIs vary along the longitudinal axis of the hippocampus. Activity within the head of the hippocampus was positively correlated with the PFC, but negatively correlates with the thalamus and PCC. The activity of the body correlated with the PCC, but negatively

Table 2

Comparison of hippocampal functional connectivity between $\mathrm{AD}$ and control subjects in relation to each target region.

\begin{tabular}{lll}
\hline & \multicolumn{1}{l}{ OC } & AD \\
\cline { 2 - 2 } & Mean \pm SD & Mean $\pm S D$ \\
\hline Left PCC & $0.294 \pm 0.031^{*}$ & $0.201 \pm 0.035$ \\
Left PFC & $0.202 \pm 0.035$ & $0.266 \pm 0.056^{*}$ \\
Left thalamus & $0.289 \pm 0.072$ & $0.312 \pm 0.112$ \\
Right PCC & $0.233 \pm 0.040$ & $0.230 \pm 0.033$ \\
Right PFC & $0.234 \pm 0.041$ & $0.243 \pm 0.054$ \\
Right thalamus & $0.316 \pm 0.076$ & $0.338 \pm 0.094$ \\
\hline
\end{tabular}

Group comparison between AD and control subjects in relation to their mean correlation coefficient of signal changes between hippocampus and each target masks. Note mean values were calculated before it was thresholded at $<0.2$. correlated with the PFC. The activity of the tail was correlated with the thalamus and PCC, but negatively correlated with the PFC. These distinguishable patterns of partial correlation of BOLD signal in which activity within a given target ROI changes synchronously with one part of the hippocampus, but anti-synchronously with another part suggests a coordinated interaction between these structures that may be important in mediating hippocampal functions.

Our approach based on using large ROI targets has the advantages of computational simplicity, while establishing the proof of principle for the methodology. However, it has at least two limitations. First, it does not formally assess the extent to which the partial correlations explain BOLD signal variation in each subregion of the hippocampus. We elected for this simplification as the explanatory power of the partial correlations is confounded by nuisance signal correlations of no interest that may vary between individuals arising from, e.g., subject movements during the scanning. Second, we used the mean signal from large, anatomically defined ROIs, rather than from individual voxels in target regions. This simplified the computational aspects of parcellation, but ignores the information that may arise from distinguishable patterns of partial connectivity of hippocampal subregions within the larger ROIs. More sophisticated approaches analogous to this have been used previously in grey matter parcellations based on diffusion tensor imaging structural connectivities (Beckmann et al., 2009) and should be explored in future studies of functional connectivity parcellations, potentially employing resting state datasets acquired over longer time periods and with greater temporal resolution (Smith et al., 2012).

\section{Anatomical correlates of the functional map of hippocampus}

Most anatomical studies of the hippocampus have been carried out in rats and some in monkey (Amaral and Cowan, 1980; Dong et al., 2009; Fanselow and Dong, 2010; Parvizi et al., 2006; van Groen et al., 1999). The ventral hippocampus in rats corresponds with the anterior, and the rat dorsal hippocampus with the posterior hippocampus in primates, including humans. Studies in rats are consistent with the general patterning identified in our functional segmentation map of the hippocampus. For example, Swanson and Cowan (Swanson and Cowan, 1977) segmented the rat hippocampus into dorsal, intermediate, and ventral parts which generally correspond with posterior, middle and anterior part of hippocampus in man. The dorsal and ventral subiculum were also defined as the parts that are dorsomedial and ventromedial to CA1, while the intermediate part was considered the portion that is the caudal end of CA1 (Kishi et al., 2000). In another study, attempts were made to divide the hippocampus into five functional segments on a flattened map along the longitudinal axis, although the exact boundaries of these domains were not very clear (Cenquizca and Swanson, 2007; Petrovich et al., 2001; Risold and Swanson, 1996). In the rat hippocampus, the dorsal part is connected with the retrosplenial area of the cingulate gyrus, the ventral part connects with visceral and somatic sensory cortices, basolateral nucleus of amygdala, agranular insular, and rostral part to prefrontal, and olfactory cortices (Cenquizca and Swanson, 2007). A single neuron recording study of awake monkeys showed an anterior-posterior heterogeneity of the hippocampal function (Colombo et al., 1998). They showed that the posterior part is more responsive to spatial tasks. 
A
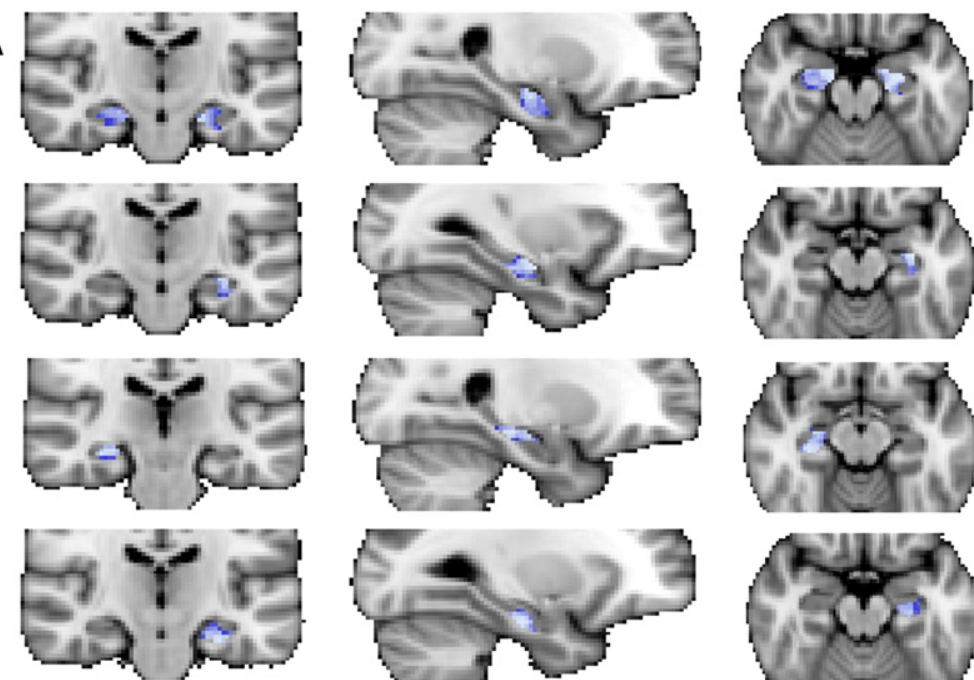

MMSE
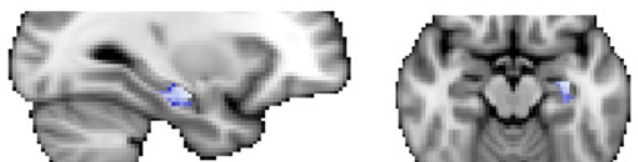

MIS
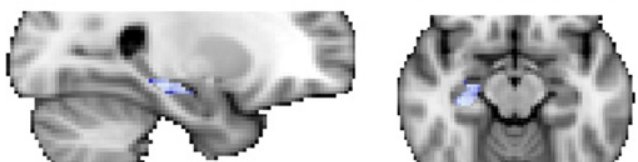

VAT
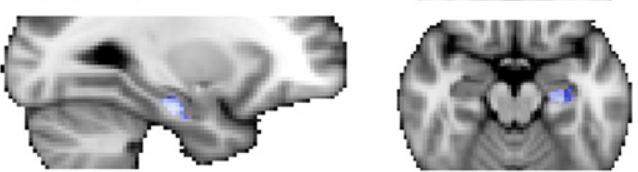

15WT
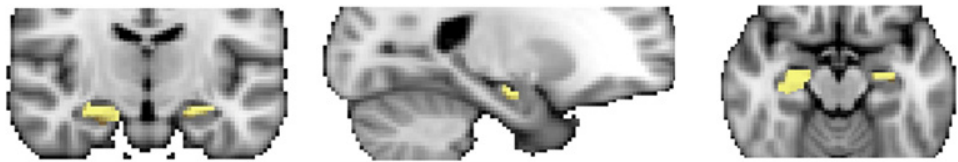

Stroop

$\mathrm{B}$
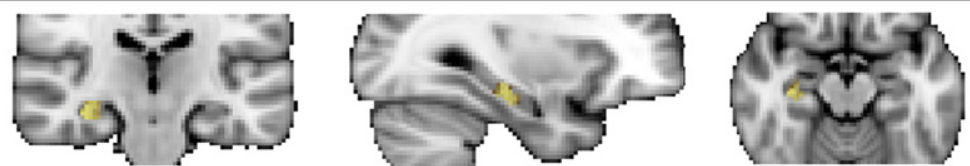

MMSE
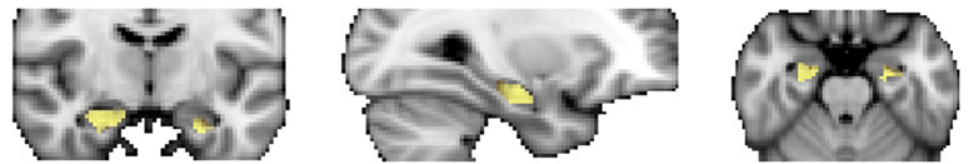

15WT
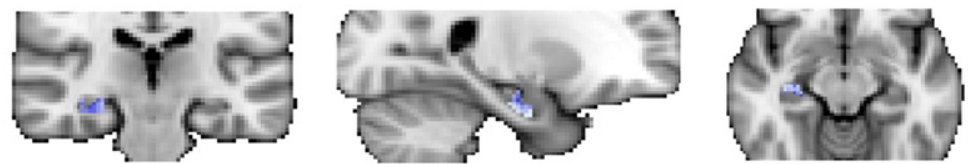

Stroop

Fig. 5. Correlation of behavioral measures with functional connectivity of hippocampus with PFC and PCC respectively. Red-yellow and blue-light blue represent positive and negative correlations respectively. All voxels have correlation coefficient of $\mathrm{r}<0.2$ (red-yellow) or $\mathrm{r}<-0.2$ (blue-light blue). Images are radiologically oriented.

Differences of functional connectivity in between the $A D$ and healthy control populations

Group comparisons of functional connectivity of the hippocampus with the target of interest showed that patients with AD had weaker connectivity with the PCC in the body of the hippocampus and stronger connectivity with the PFC in the head of the hippocampus compared to the healthy control subjects. The same relative, bidirectional change in the functional connectivity of PCC and PFC in AD patients has recently been demonstrated independently (Zhang et al., 2011).

Evidence for relative disruption of functional connectivity of the right hippocampus with the medial PFC, and PCC, as well as with the ventral anterior cingulate cortex, right inferotemporal cortex, right cuneus extending into precuneus, left cuneus, right superior and middle temporal gyrus and PCC has been shown previously (He et al., 2007; Wang et al., 2006). Others also have provided evidence of impaired hippocampus-PCC functional connectivity in AD (Greicius et al., 2004).

Diffusion tensor imaging (DTI) measures are consistent with these observations, e.g., in providing evidence that structural connectivity between PCC and hippocampus decreases in earlier stages of AD (Zhou et al., 2008). Relevant relationships between changes in the PCC and PFC are suggested by observations of decreased PCC-PFC structural connectivity in AD relative to healthy controls (Allen et al., 2007; Zhang et al., 2009). BOLD signal activity in the PCC correlates negatively with activity of the dorsolateral PFC (Fox et al., 2005). Changes of PFC-PCC, PCC-thalamus or PFC-thalamus functional connectivities thus also may affect the nature and magnitude of their partial correlations with hippocampal activity. Although the anatomical targets are defined broadly by ROIs, these definitions allow correspondences across multiple studies for a hypothesis-driven approach to characterise functional connectivities in $\mathrm{AD}$ and explore their relationships with behavioral measures. Functional connectivity to the hippocampus appears as though it may provide an additional biomarker of disease state for $\mathrm{AD}$ that may be used for diagnosing mild $A D$ or identifying those in a pre-symptomatic stage of the disease (Sorg et al., 2007). The latter will demand further study, however.

We believe that changes in functional connectivity of the hippocampus are likely to contribute to cognitive deficits in AD. Changes that we observed specifically associated with neuropsychological functions assessing episodic memory and attention. Hippocampal-PFC connectivity correlated negatively with the 15 Word Test, Visual Attention Test, Memory Impairment Screening (episodic memory) and MMSE and positively with reaction time in the Stroop test (attention, concentration and speed); greater functional connectivity between the hippocampus 

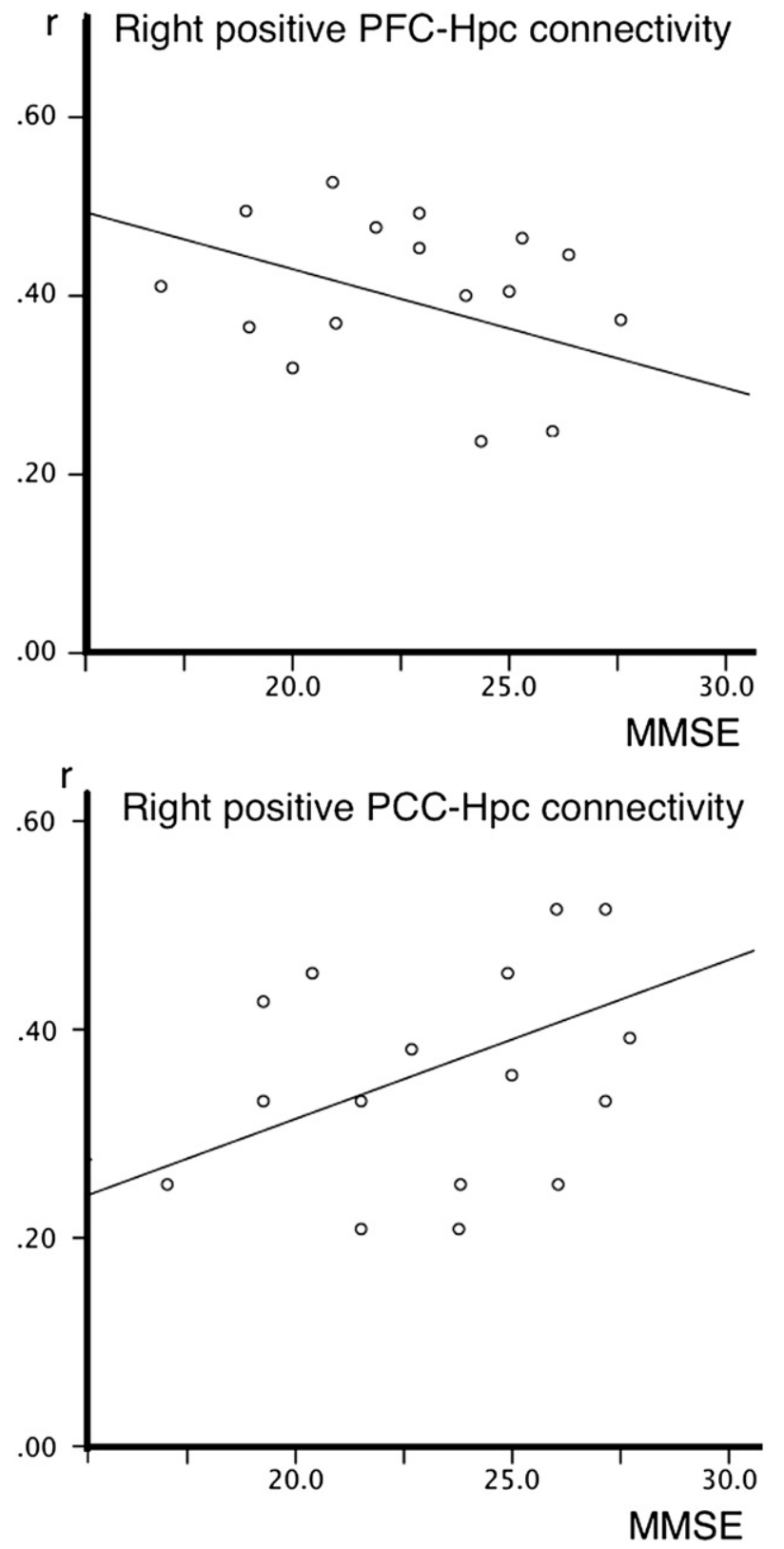

Fig. 6. Shows distribution of correlation coefficient of PFC and PPC versus MMSE in AD subjects.

and the PFC thus was related with worse cognitive performance in AD. In addition, as expected in the patient group, verbal episodic memory (15 Word Test, Memory Impairment Screening) correlated with the left and visual episodic memory (Visual Attention Test) with the right hippocampus. PCC-hippocampus functional connectivity has an opposite direction to the PFC-hippocampus functional connectivity. In addition, these functional connectivities were associated with changes in 15 Word Test (episodic memory), MMSE and Stroop test (attention, concentration and speed). Interactions between PCC and PFC, as well as with the hippocampus, may contribute to better memory and attention in AD. Changes in functional connectivity per se may reflect pathology but not in themselves be pathological, however, changes in functional connectivity also can reflect adaptive mechanisms that compensate for underlying pathology changes at microanatomical and receptor levels (Geddes et al., 1985; Scheff and Price, 2003). Distinguishing pathological and positive adaptive changes with future studies would be expected to improve the accuracy of functional connectivity as a biomarker for AD and define how functional connectivity can be used as an index of response for interventions intended to augment positive adaptive responses.

The hippocampus-PFC-thalamus-PCC network plays an important role in cognitive functions such as memory and consciousness. Anatomical studies in monkeys showed connections between the PCC and the hippocampus via the perirhinal cortex, parahippocampal gyrus and entorhinal cortex (Lavenex et al., 2002; Suzuki and Amaral, 1994). A resting state fMRI study showed that the activity of the body of the hippocampus correlated with the PCC (Zhang et al., 2009). Connections between the hippocampus and PCC are thought to play an important role in autobiographical and episodic memory (Cabeza and St Jacques, 2007; Svoboda et al., 2006).

Neuropsychological tests used in this study mainly tested memory function that is characteristically impaired in $\mathrm{AD}$. It is therefore not surprising that most neuropsychological tests correlated with the anterior half of the hippocampus which is generally associated with memory function (as opposed to, e.g., posterior half that is associated with spatial navigation). In addition, the relatively large voxel size in fMRI and small size of hippocampus mean that no comments can be made about differentiation between CA1, CA3 and dentate gyrus subregions of hippocampus.

\section{Conclusions}

We conclude that the human hippocampus consists of three functionally distinct regions in which resting state activity is correlated with activities in the thalamus, the prefrontal cortex and posterior cingulate cortex. Relative changes in the coordinated activities across this system, characterized by increased functional connectivity between the PFC and the hippocampus, and decreased functional connectivity between the PCC and the hippocampus, is seen in early AD. These changes correlated with worse performance on cognitive tests in $\mathrm{AD}$, suggesting that these functional connectivities may provide specific indices for assessing memory functions and their changes in $\mathrm{AD}$. We suggest that this method of hippocampal functional segmentation could be of use in early diagnosis of $\mathrm{AD}$ and possibly in monitoring the effects of interventions to improve memory functions or slow the progression of pathology.

\section{References}

Allen, G., Barnard, H., McColl, R., Hester, A.L., Fields, J.A., Weiner, M.F., Ringe, W.K., Lipton, A.M., Brooker, M., McDonald, E., Rubin, C.D., Cullum, C.M., 2007. Reduced hippocampal functional connectivity in Alzheimer disease. Arch. Neurol. 64, 1482-1487.

Amaral, D.G., Cowan, W.M., 1980. Subcortical afferents to the hippocampal formation in the monkey. J. Comp. Neurol. 189, 573-591.

Andersson, J.L.R., Jenkinson, M., Smith, M., 2007. Non-linear registration, aka spatial normalisation. FMRIB technical report TR07JA2 from http://www.fmrib.ox.ac.uk/ analysis/techrep.

Beckmann, C.F., Smith, S.M., 2004. Probabilistic independent component analysis for functional magnetic resonance imaging. IEEE Trans. Med. Imaging 23, 137-152.

Beckmann, C.F., DeLuca, M., Devlin, J.T., Smith, S.M., 2005. Investigations into resting-state connectivity using independent component analysis. Philos. Trans. R. Soc. Lond. B Biol. Sci. 360, 1001-1013.

Beckmann, M., Johansen-Berg, H., Rushworth, M.F., 2009. Connectivity-based parcellation of human cingulate cortex and its relation to functional specialization. J. Neurosci. 29, 1175-1190.

Biswal, B., Yetkin, F.Z., Haughton, V.M., Hyde, J.S., 1995. Functional connectivity in the motor cortex of resting human brain using echo-planar MRI. Magn. Reson. Med. 34, 537-541.

Cabeza, R., St Jacques, P., 2007. Functional neuroimaging of autobiographical memory. Trends Cogn. Sci. 11, 219-227.

Carmichael, S.T., Price, J.L., 1995. Limbic connections of the orbital and medial prefronta cortex in macaque monkeys. J. Comp. Neurol. 363, 615-641.

Cenquizca, L.A., Swanson, L.W., 2007. Spatial organization of direct hippocampal field CA1 axonal projections to the rest of the cerebral cortex. Brain Res. Rev. 56, 1-26.

Colombo, M., Fernandez, T., Nakamura, K., Gross, C.G., 1998. Functional differentiation along the anterior-posterior axis of the hippocampus in monkeys. J. Neurophysiol. 80, 1002-1005.

Cordes, D., Haughton, V.M., Arfanakis, K., Carew, J.D., Turski, P.A., Moritz, C.H., Quigley M.A., Meyerand, M.E., 2001. Frequencies contributing to functional connectivity in the cerebral cortex in "resting-state" data. AJNR Am. J. Neuroradiol. 22, 1326-1333.

Damoiseaux, J.S., Smith, S.M., Witter, M.P., Sanz-Arigita, E.J., Barkhof, F., Scheltens, P., Stam, C.J., Zarei, M., Rombouts, S.A., 2009. White matter tract integrity in aging and Alzheimer's disease. Hum. Brain Mapp. 30, 1051-1059. 
Dong, H.W., Swanson, L.W., Chen, L., Fanselow, M.S., Toga, A.W., 2009. Genomic-anatomic evidence for distinct functional domains in hippocampal field CA1. Proc. Natl. Acad. Sci. U. S. A. 106, 11794-11799.

Fanselow, M.S., Dong, H.W., 2010. Are the dorsal and ventral hippocampus functionally distinct structures? Neuron $65,7-19$.

Fazekas, F., Chawluk, J.B., Alavi, A., Hurtig, H.I., Zimmerman, R.A., 1987. MR signal abnormalities at $1.5 \mathrm{~T}$ in Alzheimer's dementia and normal aging. AJR Am. J. Roentgenol. 149, 351-356.

Fox, M.D., Snyder, A.Z., Vincent, J.L., Corbetta, M., Van Essen, D.C., Raichle, M.E., 2005. The human brain is intrinsically organized into dynamic, anticorrelated functional networks. Proc. Natl. Acad. Sci. U. S. A. 102, 9673-9678.

Geddes, J.W., Monaghan, D.T., Cotman, C.W., Lott, I.T., Kim, R.C., Chui, H.C., 1985. Plasticity of hippocampal circuitry in Alzheimer's disease. Science 230, 1179-1181.

Genovese, C.R., Lazar, N.A., Nichols, T., 2002. Thresholding of statistical maps in functional neuroimaging using the false discovery rate. Neuroimage $15,870-878$.

Greicius, M.D., Krasnow, B., Boyett-Anderson, J.M., Eliez, S., Schatzberg, A.F., Reiss, A.L. Menon, V., 2003a. Regional analysis of hippocampal activation during memory encoding and retrieval: fMRI study. Hippocampus 13, 164-174.

Greicius, M.D., Krasnow, B., Reiss, A.L., Menon, V., 2003b. Functional connectivity in the resting brain: a network analysis of the default mode hypothesis. Proc. Natl. Acad. Sci. U. S. A. 100, 253-258.

Greicius, M.D., Srivastava, G., Reiss, A.L., Menon, V., 2004. Default-mode network activity distinguishes Alzheimer's disease from healthy aging: evidence from functional MRI Proc. Natl. Acad. Sci. U. S. A. 101, 4637-4642.

Greicius, M.D., Supekar, K., Menon, V., Dougherty, R.F., 2009. Resting-state functional connectivity reflects structural connectivity in the default mode network. Cereb. Cortex 19, 72-78.

He, Y., Wang, L., Zang, Y., Tian, L., Zhang, X., Li, K., Jiang, T., 2007. Regional coherence changes in the early stages of Alzheimer's disease: a combined structural and resting-state functional MRI study. Neuroimage 35, 488-500.

Insausti, R., Amaral, D.G., Cowan, W.M., 1987. The entorhinal cortex of the monkey: III. Subcortical afferents. J. Comp. Neurol. 264, 396-408.

Jenkinson, M., Smith, S., 2001. A global optimisation method for robust affine registration of brain images. Med. Image Anal. 5, 143-156.

Kahn, I., Andrews-Hanna, J.R., Vincent, J.L., Snyder, A.Z., Buckner, R.L., 2008. Distinct cortical anatomy linked to subregions of the medial temporal lobe revealed by intrinsic functional connectivity. J. Neurophysiol. 100, 129-139.

Kishi, T., Tsumori, T., Ono, K., Yokota, S., Ishino, H., Yasui, Y., 2000. Topographical organization of projections from the subiculum to the hypothalamus in the rat. J. Comp. Neurol. 419, 205-222.

Lavenex, P., Suzuki, W.A., Amaral, D.G., 2002. Perirhinal and parahippocampal cortices of the macaque monkey: projections to the neocortex. J. Comp. Neurol. 447, 394-420.

Maguire, E.A., Frackowiak, R.S., Frith, C.D., 1997. Recalling routes around london: activation of the right hippocampus in taxi drivers. J. Neurosci. 17, 7103-7110.

McKhann, G., Drachman, D., Folstein, M., 1984. Clinical diagnosis of Alzheimer's disease: Report of the NINCDS-ADRDA work group under the auspices of Department of Health and Human Services Task Force on Alzheimer's disease. Neurology 34, 939-944.

Mesulam, M.M., Van Hoesen, G.W., Pandya, D.N., Geschwind, N., 1977. Limbic and sensory connections of the inferior parietal lobule (area PG) in the rhesus monkey: a study with a new method for horseradish peroxidase histochemistry. Brain Res. 136, 393-414.

Morris, R., Petrides, M., Pandya, D.N., 1999. Architecture and connections of retrosplenia area 30 in the rhesus monkey (Macaca mulatta). Eur. J. Neurosci. 11, 2506-2518.

Moser, M.B., Moser, E.I., 1998. Functional differentiation in the hippocampus. Hippocampus 8, 608-619.

Nichols, T.E., Holmes, A.P., 2002. Nonparametric permutation tests for functional neuroimaging: a primer with examples. Hum. Brain Mapp. 15, 1-25.

O'Reilly, J.X., Beckmann, C.F., Tomassini, V., Ramnani, N., Johansen-Berg, H., 2009. Distinct and overlapping functional zones in the cerebellum defined by resting state functional connectivity. Cereb. Cortex 20, 953-965.
Parvizi, J., Van Hoesen, G.W., Buckwalter, J., Damasio, A., 2006. Neural connections of the posteromedial cortex in the macaque. Proc. Natl. Acad. Sci. U. S. A. 103, 1563-1568. Petrovich, G.D., Canteras, N.S., Swanson, L.W., 2001. Combinatorial amygdalar inputs to hippocampal domains and hypothalamic behavior systems. Brain Res. Brain Res. Rev. 38, 247-289.

Raichle, M.E., 2010. Two views of brain function. Trends Cogn. Sci. 14, 180-190.

Raichle, M.E., MacLeod, A.M., Snyder, A.Z., Powers, W.J., Gusnard, D.A., Shulman, G.L., 2001. A default mode of brain function. Proc. Natl. Acad. Sci. U. S. A. 98, 676-682.

Risold, P.Y., Swanson, L.W., 1996. Structural evidence for functional domains in the rat hippocampus. Science 272, 1484-1486.

Scheff, S.W., Price, D.A., 2003. Synaptic pathology in Alzheimer's disease: a review of ultrastructural studies. Neurobiol. Aging 24, 1029-1046.

Smith, S.M., Jenkinson, M., Woolrich, M.W., Beckmann, C.F., Behrens, T.E., Johansen-Berg, H., Bannister, P.R., De Luca, M., Drobnjak, I., Flitney, D.E., Niazy, R.K., Saunders, J., Vickers, J., Zhang, Y., De Stefano, N., Brady, J.M., Matthews, P.M., 2004. Advances in functional and structural MR image analysis and implementation as FSL. Neuroimage 23 (Suppl. 1), S208-219.

Smith, S.M., Miller, K.L., Moeller, S., Xu, J., Auerbach, E.J., Woolrich, M.W., Beckmann, C.F., Jenkinson, M., Andersson, J., Glasser M.F, Van Essen, D.C. Feinberg D. A. Yacoub, E.S, Ugurbil, K., 2012. Temporally-independent functional modes of spontaneous brain activity. Proc. Natl. Acad. Sci. U. S. A. 109, 3131-3136.

Sorg, C., Riedl, V., Muhlau, M., Calhoun, V.D., Eichele, T., Laer, L., Drzezga, A., Forstl, H., Kurz, A., Zimmer, C., Wohlschlager, A.M., 2007. Selective changes of resting-state networks in individuals at risk for Alzheimer's disease. Proc. Natl. Acad. Sci. U. S. A. 104, 18760-18765.

Stein, T., Moritz, C., Quigley, M., Cordes, D., Haughton, V., Meyerand, E., 2000. Functional connectivity in the thalamus and hippocampus studied with functional MR imaging. AJNR Am. J. Neuroradiol. 21, 1397-1401.

Suzuki, W.A., Amaral, D.G., 1994. Perirhinal and parahippocampal cortices of the macaque monkey: cortical afferents. J. Comp. Neurol. 350, 497-533.

Svoboda, E., McKinnon, M.C., Levine, B., 2006. The functional neuroanatomy of autobiographical memory: a meta-analysis. Neuropsychologia 44, 2189-2208.

Swanson, L.W., Cowan, W.M., 1977. An autoradiographic study of the organization of the efferent connections of the hippocampal formation in the rat. J. Comp. Neurol. $172,49-84$.

van Groen, T., Kadish, I., Wyss, J.M., 1999. Efferent connections of the anteromedial nucleus of the thalamus of the rat. Brain Res. Brain Res. Rev. 30, 1-26.

Wang, L., Zang, Y., He, Y., Liang, M., Zhang, X., Tian, L., Wu, T., Jiang, T., Li, K., 2006. Changes in hippocampal connectivity in the early stages of Alzheimer's disease: evidence from resting state fMRI. Neuroimage 31, 496-504.

Zarei, M., Patenaude, B., Damoiseaux, J., Morgese, C., Smith, S., Matthews, P.M., Barkhof, F., Rombouts, S.A., Sanz-Arigita, E., Jenkinson, M., 2010. Combining shape and connectivity analysis: an MRI study of thalamic degeneration in Alzheimer's disease. Neuroimage 49, 1-8.

Zhang, Y., Brady, M., Smith, S., 2001. Segmentation of brain MR images through a hidden Markov random field model and the expectation-maximization algorithm. IEEE Trans. Med. Imaging 20, 45-47.

Zhang, D., Snyder, A.Z., Fox, M.D., Sansbury, M.W., Shimony, J.S., Raichle, M.E., 2008. Intrinsic functional relations between human cerebral cortex and thalamus. J. Neurophysiol. $100,1740-1748$.

Zhang, H.Y., Wang, S.J., Xing, J., Liu, B., Ma, Z.L., Yang, M., Zhang, Z.J., Teng, G.J., 2009. Detection of PCC functional connectivity characteristics in resting-state fMRI in mild Alzheimer's disease. Behav. Brain Res. 197, 103-108.

Zhang, H.Y., Wang, S.J., Liu, B., Ma, Z.L., Yang, M., Zhang, Z.J., Teng, G.J., 2011. Resting brain connectivity: changes during the progress of Alzheimer disease. Radiology 256, 598-606.

Zhou, Y, Dougherty Jr. J.H., Hubner, K.F., Bai, B. Cannon, R.L., Hutson, R.K., 2008. Abnormal connectivity in the posterior cingulate and hippocampus in early Alzheimer's disease and mild cognitive impairment. Alzheimers Dement. 4, 265-270. 